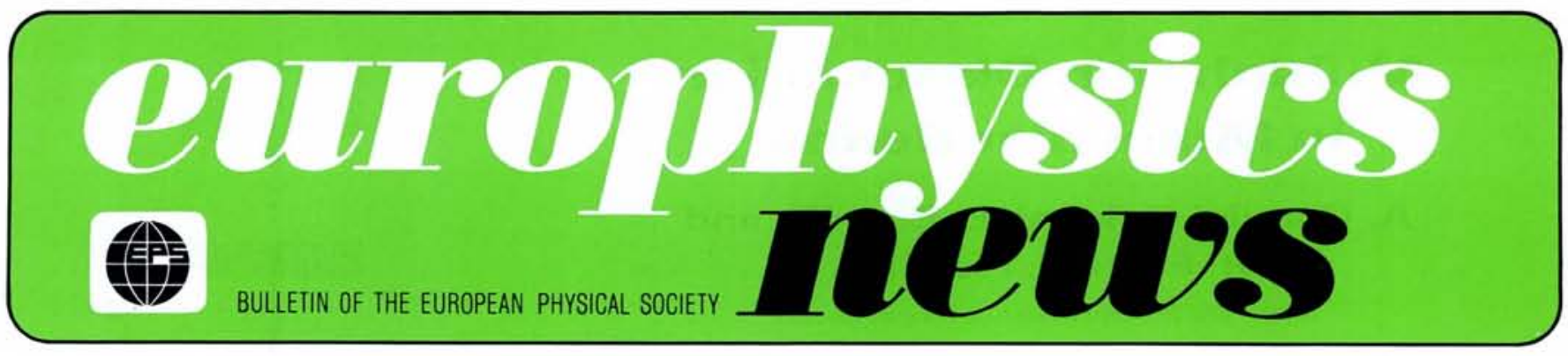

J.A.

\title{
Atomic and Molecular Physics
}

\section{An Exciting Field of Many Facets}

Several years ago a well-known physicist wrote that through the Schrödinger equation, all information on atoms and molecules could easily be obtained provided one had the time and patience to solve the equation for a specific case. The implication was that one had moved away from research in fundamental physics into routine "stamp collection" — to paraphrase another famous old dictum.

Moreover, since the study of atomic and molecular properties must draw upon different and often quite distinct fields of science, it was accused of falling between stools: experiments may be aimed at detecting one specific and often very small effect, or they may aim at providing broad generalisations only possible after looking at many similar measurements on a large number of systems. As studies are often at the boundary separating well-established disciplines they were deemed liable to make only marginal contributions to the larger territories of knowledge they attempted to unite.

The series of articles which follows proves how false these predictions were. In reality the broad and lively field of atomic and molecular physics continues to un cover fundamental aspects of the laws of physics. Even the most superficial observer must have noticed that the last ten years or so have witnessed a tremendous growth in our knowledge, both theoretical and experimental, of the behaviour of atoms and molecules under very different conditions, and of the detailed mechanisms which preside over their microscopic interactions. As several authors here indicate, atomic and molecular physics has become one of the areas of physics research where the collaboration between theorists and experimentalists has been most intimate, something that is difficult to achieve in other branches of research where the sheer size and time scale of experiments precludes theory from steering or quickly redirecting experimental efforts. It is a tradition of the atomic and molecular sciences that those participating in a given area of research should understand as far as possible all the relevant aspects of the problem. Making measurements, working out the mathematical foundations, devising numerical models..., these are often done by the same people, which leads to a very special sense of involvement and satisfaction.

The topics chosen for this series are typical of areas which have generated great excitement as one finally understands the wonderful simplicity of the causes of complicated phenomena or one sees effects that had previously remained elusive. For example, it was evident that a strong connexion existed between the evolution of stars and the manifestation of strong and weak forces in nuclear phenomena; astrophysics and elementary particle physics have often been dealing with the same problems in recent years. Less known is that atomic phenomena play a rather large role in both fields, and that theoretical advances in atomic physics have helped to explain the most diverse observations. The articles on atomic astrophysics and on atomic parity non-conservation show how deeply atomic data collecting and analysis have delved into the basic areas of interest to both sectors.

Molecular linear spectroscopy has been the staple diet of chemical research for many years, but the development of lasers as common laboratory tools has given access to many new and previously undreamed of effects which involve molecular energy levels. We can, for instance, look at molecular energy redistribution and storage in detail, observe the structural properties of weak, near-chemical species, study directly the structures of that elusive species which are the cornerstone of chemical reactions, the transition state or activated complex. The contributions on multiphoton dissociation on dimers, and molecular resonances discuss the novel ways employed today to look at the bound states of atoms with many electrons: here again all the forces involved have been known for a long time, but their manifestation still brings surprises on an almost daily basis.

New experiments on energetic atomic collisions have spurred renewed theoretical efforts on the well known problem of Coulomb forces in a three-body system and have made us far more aware of the variety of ways in which an old puzzle can be studied and solved, thus providing better explanations for observed phenomena. It will be seen that there is yet much to be learned about Coulomb forces.

Space limitations oblige us to omit other aspects of atomic and molecular physics but it is hoped that we have demonstrated that solving the Schrödinger equation is still a worthwhile task, very much science and not by any means stamp collecting. The gathering of several hundred physicists from all over Europe at the Second European Conference on Atomic and Molecular Physics (ECAMP II) in Amsterdam in April 1985 is confirmation that the field is alive and attractive to an ever increasing number of researchers.

\section{F.A. Gianturco, Rome}

Chairman, Atomic and Molecular Physics Division

\section{Contents}

Atomic and Molecular Physics

Multiphoton Dissociation in

Molecular Beams

Theory of Energetic lon-Atom Collisions

Vibrational Predissociation and Dimer Spectroscopy

Atomic Processes in Astronomy

Parity Non-conservation in Heavy Atoms

Theory of Resonances in Molecular Systems

1985 Hewlett-Packard Europhysics Prize

5th CMD General Conference see Feb. see Feb.

9 (2)

Deneral Conference 